\title{
SARS-CoV-2 antibodies in breastmilk after COVID-19 and/or vaccination
}

\author{
Cristina VANCSA \\ "Sf. Ioan" Hospital, Bucharest, Romania
}

\begin{abstract}
Several studies to date show that most people who have been infected with SARS-CoV-2 or vaccinated develop antibodies specific to this virus. However, the levels of these antibodies can vary between those who have severe disease (higher levels of antibodies) and those with milder disease or asymptomatic infection (lower levels of antibodies). Many studies are underway to better understand the levels of antibodies that are needed for protection, and how long these antibodies last.

In April 2020, scientists discovered that antibodies can be transmitted through breast milk. Study results suggest that passive acquisition of antibodies against SARS-CoV-2 is realized by ingesting breast milk.

New research published on April 2021 suggests that nursing mothers who receive a COVID-19 vaccine may also protect their babies from the virus.
\end{abstract}

Keywords: breastfeeding, breastmilk, COVID-19, human milk, SARS-CoV-2, antibodies, vaccine

\section{INTRODUCTION}

SARS-CoV-2 is a virus of the species severe acute respiratory syndrome-related coronavirus (SARSr-CoV) [1]. It is believed to have zoonotic origins and has close genetic similarity to bat coronaviruses, suggesting it emerged from a bat-borne virus [2,3].

Coronavirus 2019 (COVID-19) is a contagious disease caused by severe acute respiratory syndrome coronavirus 2 (SARS-CoV-2). The first known case was identified in Wuhan, China, in December 2019 [4]. The disease has since spread worldwide, leading to an ongoing pandemic $[5,6]$.

Symptoms of COVID-19 are variable, but often include fever, cough, headache, fatigue, breathing difficulties, and loss of smell and taste [7]. Symptoms may begin one to fourteen days after exposure to the virus [1]. At least a third of those who are infected do not develop noticeable symptoms. Multi-year studies are underway to further investigate the long-term effects of the disease [7].
People who are infected can transmit the virus to others up to two days before they show symptoms themselves. It is similar in case of those who do not experience symptoms. People remain infectious for up to ten days after the onset of symptoms in moderate cases and up to twenty days in severe cases [8].

Although work is underway to develop drugs that inhibit the virus, the primary treatment is symptomatic. Management involves the treatment of symptoms, supportive care, isolation, and experimental measures [9].

\section{DEFINITIONS}

An antibody (Ab) [10], also known as an immunoglobulin (Ig) [11], is a large, Y-shaped protein used by the immune system to identify and neutralize foreign objects such as pathogenic bacteria and viruses. The antibody recognizes a unique molecule of the pathogen, called an antigen [12]. An antibody can tag a mi- 
crobe or an infected cell for attack by other parts of the immune system, or can neutralize it directly (for example, by blocking a part of a virus that is essential for its invasion).

Antibodies work by recognizing and sticking to specific proteins, such as those found on the surfaces of viruses and bacteria, in a highly specific way. When the body encounters a microbe for the first time, immune cells produce antibodies that specifically recognize proteins associated with that particular microbe.

After recovering from an infection or receiving a vaccine, a small number of these antibody-producing immune cells usually remain in the body as memory cells, providing immunity to future infections with the same bug. Because memory cells and antibodies are already present, next time the body encounters the same microbe, the immune response is much faster and can stop the infection from taking hold [13].

\section{COVID-19 TYPES OF ANTIBODIES}

- IgM antibodies, which develop early in an infection

- IgG antibodies, which develop a few weeks postinfection and are key in establishing immunity after infection

- IgA antibodies, which protect the surfaces of mucous membranes throughout the body.

There are many studies underway to better understand the antibody response following infection to SARS-CoV-2 [1]. Several studies to date show that most people who have been infected with SARS-CoV-2 develop antibodies specific to this virus [1]. However, the levels of these antibodies can vary between those who have severe disease (higher levels of antibodies) and those with milder disease or asymptomatic infection (lower levels of antibodies). Many studies are underway to better understand the levels of antibodies that are needed for protection, and how long these antibodies last.

To date, there are reports of individuals who have been reinfected with SARS-CoV-2 [14]. There are likely to be more examples of reinfection reported and scientists are working to understand the role of the immune response in the first and second infection.

\section{VACCINATION}

Romania has currently authorized the use of four different COVID-19 vaccines made by Pfizer-BioNTech, Moderna, Vaxzervia (AstraZeneca), and Janssen (Johnson \& Johnson's vaccine division) [15].

The Pfizer-BioNTech [16] and Moderna [17] vaccines use mRNA technology while the AstraZeneca [18] and single-dose J\&J [19] shots are considered viral vector-based vaccines.
Viral vector-based vaccines, such as those developed by AstraZeneca and Johnson \& Johnson, use a harmless virus, or adenovirus, as a delivery system to trigger the immune system to create antibodies to fight off an infection by SARS-CoV-2, which is the virus that causes COVID-19 [18,19].

The adenovirus is not SARS-CoV- 2 itself, but rather a different, harmless virus that has been manipulated so it's unable to replicate and cause illness.

In the case of COVID-19, the vector virus delivers specific genetic instructions to the cells in the body to produce a harmless piece of SARS-CoV-2 called the spike protein. The cells then display this spike protein and the immune system triggers a response.

As a result, the immune system produces antibodies to the specific spike protein in order to fight off what it thinks is an infection. If the immune system encounters the real SARS-CoV-2 virus and its spike proteins, it will already be prepared to launch a defence against it.

The viral vector-based vaccines are genetically modified so they're unable to replicate, which means once the antibodies are created, the viral vector is cleared for good.

For their COVID-19 vaccines, Pfizer-BioNTech and Moderna use a novel technology that has never been approved for widespread use before the pandemic $[16,17]$.

While there are similarities in how both mRNA and viral vector-based vaccines instruct cells to create the SARS-CoV-2 spike protein, mRNA vaccines differ in that they don't contain any live virus.

The viral vector-based COVID-19 vaccines developed by AstraZeneca [18] and Johnson \& Johnson [19] have been linked to an extremely rare and potentially life-threatening blood-clotting syndrome called vaccine-induced thrombotic thrombocytopenia (VITT) which is the combination of low platelet counts with blood clots. The risk for developing this syndrome is estimated to be anywhere from one case in 100,000 doses to one case in 250,000 [20].

The vaccine contains mRNA, which is a messenger that codes for various proteins, but is never incorporated into the DNA (genetic code) of cells. In addition, the life of a messenger RNA as contained in the Pfizer and Moderna vaccines is only a few hours inside the body [21].

\section{COMPARING THE COVID-19 VACCINES}

[22]

Pfizer 95\% efficacy in preventing COVID-19 in those without prior infection. In early May, the Pfizer-BioNTech vaccine was found to be more than $95 \%$ effective against severe disease or death from the variants first 
detected in the United Kingdom (B.1.1.7) and South Africa (B.1.351) in two studies based on real-world use of the vaccine. While the efficacy against infection varied between the two studies, both also showed the vaccine provides strong protection.

Moderna 94.1\% effective at preventing symptomatic infection in people with no evidence of previous COVID-19 infection. Some research has suggested that Moderna's vaccine may provide protection against the variants of the virus. Researchers are still studying this.

Astra Zeneca76\% effective at preventing symptomatic infection in people with no evidence of previous COVID-19 infection. So far it seems to work better against the mutation that emerged in Great Britain than the one that emerged in South Africa.

J\&J $72 \%$ overall efficacy. Some research has suggested protection against variants.

\section{SARS-COV-2 ANTIBODIES AND BREAST MILK}

\section{Newborns won't get COVID through infected mom's breast milk}

Jerusalem Hospital published the results of their study: researchers followed 55 infants born at the Israeli medical center to mothers who tested positive for SARS-CoV-2. All of the newborns tested negative for the infection soon after delivery [23].

Three-quarters of the babies were given breast milk during their hospital stay, and even more $-85 \%$ - were breastfed after going home. None became infected with the coronavirus, based on screening tests done two to three weeks after leaving the hospital.

Earlier in the pandemic, the Jerusalem Hospital had a policy of separating newborns from their SARS-CoV-2-positive moms. Because of that, infants in this study were given pumped breast milk by bottle.

The study shows this is no longer necessary, as long as safeguards like mask-wearing and hand-washing are followed.

\section{Breast milk harbors antibodies to SARS-CoV-2}

Rebecca Powell, immunologist at the School of Medicine at Mount Sinai in New York City, discovered in April 2020 that antibodies can be transmitted through breast milk [24].

One important feature of these antibodies, whether specific to the virus or not, is that they are secretory antibodies. The B cells that secrete antibodies into milk originate from the mucosal immune system in the mother's small intestine. Those B cells travel through the blood to the mammary glands and secrete IgA that's then shuttled from the mammary tissue to the milk via a transporter protein. Those proteins, leave pieces of themselves on the antibodies, wrapping around them and protecting them from being degraded in the infant mouth and gut.

That difference could give breast milk-derived antibodies an advantage over blood-based ones as far as therapeutic options go. Antibodies such as IgG that are extracted from serum and transfused into the blood of a sick person travel throughout the body and might not go where they are needed. But secretory antibodies, such as IgA from breast milk, could be extracted and then inhaled into the respiratory tract - just where those antibodies are needed in COVID-19.

Research suggests that blood-derived antibodies can last months. Secretory antibodies in breast milk might last longer, Powell's most recent data indicate, and that means there could be a longer window to collect antibodies from lactating donors after they've recovered from COVID-19.

Also, a study in China discovered that IgM and IgG antibodies to SARS-CoV-2 were detected in breast milk, cord blood and neonatal serum. The study results suggest that passive acquisition of antibodies against SARS-CoV-2 is available by ingesting breast milk [25].

\section{ANTIBODIES AND VACCINATION}

Nursing mothers who receive a COVID-19 vaccine may pass protective antibodies to their babies through breast milk for at least 80 days following vaccination, suggests new research from Washington University School of Medicine in St. Louis [26].

Published in the American Journal of Obstetrics and Gynecology, the study tracked levels of COVID-19 antibodies in breast milk from a baseline before the mothers' first vaccinations and on a weekly basis for 80 days after those initial vaccinations with Pfizer.

While other recent research has shown that COVID-19 vaccines generate antibodies that are passed to nursing infants through breast milk, this is thought to be the first study to track specific levels of these antibodies in breast milk over an extended time period.

The babies of women included in the study ranged in age from one month to 24 months old. To gauge immune response in the breast milk, researchers monitored levels of the immunoglobulins IgA and IgG, which are antibodies deployed by the immune system to fight infections in babies.

While further studies of maternal COVID-19 vaccination are needed to characterize the length of antibody production in breast milk and the effect on infant infection rates, recent research continues to confirm that the COVID-19 vaccine offers real benefits for protecting both mother and child.

A recent study published on May 4th 2021 was conducted in Israel, where a national COVID-19 vaccina- 
tion program began on December 20, 2020, and prioritized breastfeeding women belonging to vaccine target groups [27].

In total, investigators collected 504 breast milk samples from the 84 women who completed the study. Breastfeeding women vaccinated against COVID-19 had elevated levels of antibodies against SARS-CoV-2 in their breast milk as soon as 2 weeks after their first vaccination dose.

Though investigators in this study did not perform functional assays, previous studies reported strong neutralizing effects of antibodies to COVID-19 in breast milk. This implies that infants of mothers with antiSARS-CoV-2 antibodies in their breast milk are potentially protected against COVID-19.

A study published in American Journal of Obstetrics and Gynecology concluded that COVID-19 mRNA vaccines generated robust humoral immunity in pregnant and lactating women. Vaccine-induced immune responses were significantly greater than the response to natural infection. Immune transfer to neonates occurred via placenta and breast milk [28].

On March 14th 2021, University of Minnesota released a study that assessed immune response in a convenience sample of 103 pregnant, lactating, and nonpregnant women given either the Pfizer/BioNTech (54\%) or the Moderna (46\%) COVID-19 vaccine from December 2020 through March 2021 [29].

Data suggest that there may be greater cross-reactivity for functional nonneutralizing antibodies and cellular immune responses than for neutralizing antibodies against SARS-CoV-2 variants of concern.

The results are important, the researchers said, because pregnant women with symptomatic COVID-19 are at higher risk for death, intensive care unit admission, and mechanical ventilation than their nonpregnant peers. Likewise, preterm births and stillbirths have been noted in cases of maternal COVID-19 infection, although maternal-fetus coronavirus transmission has been rare, and newborns gain passive immunity via placental antibody transfer and breast milk after natural maternal infection.

The research from Washington University School of Medicine in St. Louis suggests also that nursing mothers who receive a COVID-19 vaccine may also protect their babies from the virus [26]. The researchers found a major boost in antibodies against the COVID-19 virus in breast milk starting two weeks after vaccination, and this response was sustained for the course of the study, which was almost three months long.

Study showed a boost in antibodies against the COVID-19 virus in breast milk starting two weeks after the first shot (Pfizer), and this response was sustained for almost three months long. The antibodies levels were still high at the end of our study, so the protection likely extends even longer.
These types of antibodies coat babies mouths and throats and protect against disease when a baby is drinking breast milk. Vaccination while breastfeeding not only protects the mother, but also could protect the infant.

\section{UNINTENDED CONSEQUENCES}

After getting a COVID-19 vaccine, women are selling their breast milk online advertising the benefits related to SARS-CoV-2 infection. A 2013 study found high levels of overall bacterial growth and frequent contamination with pathogenic bacteria in breast milk samples bought through the Internet. Although raw milk contains antibodies, it could also transmit pathogens, including HIV [30]. Milk banks uses donated breast milk which is pooled together and pasteurized, destroying pathogens, but not the antibodies.

\section{FURTHER RESEARCH}

Small children are not yet eligible to receive any of the available vaccines and likely will not be eligible for some time, however they are still at risk of contracting acute COVID-19 illness. While young children have not been a primary age group for severe illness or death, infants and young children can develop a severe inflammatory illness known as MIS-C (multisystem inflammatory syndrome in children), as a manifestation of post-coronavirus illness. Children with this syndrome present with high fever, fatigue, rash, myalgias (muscle pain), and occasionally cardiac complications. Many require hospitalization, and some have succumbed to complications secondary to MIS-C. This entity tends to be more common in infants and toddlers, who will be the last age groups to obtain approval to receive a vaccine [31].

Rebecca Powell, professor at Mt. Sinai, whose research focuses on infectious diseases and human milk for the past years, concluded that as a child grows the milk itself doesn't change in its dosage of antibodies, but in wealthy countries, babies are usually taking in less. The frequency of the feedings increases the efficacy of antibody passive acquisition through breast milk [24].

In an exploratory analysis published by The Lancet on May 13, 2021, receipt of a COVID-19 mRNA vaccine was found immunogenic in pregnant women [32].

Clinical trials for both the Pfizer-BioNTech BNT162b2 and Moderna mRNA-1273 vaccines demonstrated the ability to prevent infection and severe disease [33].

\section{CONCLUSIONS}

Women who get vaccinated against COVID-19 or are infected with SARS-CoV-2 while breastfeeding pass 
antibodies to their children through their milk. Breastmilk antibodies are different in both function and composition. Rather than enter the bloodstream, these antibodies aim to especially protect the entry points to the body. They target what we call mucosal tissue the respiratory tract, oral cavity, gut lining. In babies, breastfeeding may be protective due to protective antibodies passed from the immune mother to the infant, conferring passive immunity.
Vaccine-induced immune responses were significantly greater than the response to natural infection. Further studies of maternal COVID-19 vaccination are needed to characterize the length of antibody production in breast milk and the effect on infant infection rates.

\section{Conflict of interest: none declared Financial support: none declared}

\section{REFERENCES}

1. Coronaviridae Study Group of the International Committee on Taxonomy of Viruses. The species Severe acute respiratory syndrome-related coronavirus: classifying 2019-nCoV and naming it SARS-CoV-2. Nat Microbiol. 2020;5;536-544.

2. Andersen KG, Rambaut A, Lipkin WI, Holmes EC, Garry RF. The proximal origin of SARS-CoV-2. Nat Med. 2020 Apr; 26(4):450-452.

3. Naming the coronavirus disease (COVID-2019) and the virus that causes it. World Health Organization. Archived from the original on 28 February 2020. Retrieved 14 December 2020.

4. Zhou P, Yang XL, Wang XG, Hu B, Zhang L, et al. A pneumonia outbreak associated with a new coronavirus of probable bat origin. Nature. 2020 Mar;579(7798):270-273.

5. Hui M Why won't the WHO call the coronavirus by its name, SARS-CoV-2?. Quartz. Archived from the original on 25 March 2020.

6. Statement on the second meeting of the International Health Regulations (2005) Emergency Committee regarding the outbreak of novel coronavirus (2019-nCoV). World Health Organization (WHO) (Press release). 30 January 2020.

7. Agyeman $A A, C h i n ~ K L$, Landersdorfer $C B$, Liew D, Ofori-Asenso R. Smell and Taste Dysfunction in Patients With COVID-19: A Systematic Review and Meta-analysis. Mayo Clin Proc. 2020 Aug;95(8):1621-1631.

8. Coronavirus Disease 2019 (COVID19). Centers for Disease Control and Prevention. Archived from the original on 11 February 2020. Retrieved 6 December 2020.

9. Siemieniuk RA, Bartoszko JJ, Ge L, Zeraatkar D, et al. Drug treatments for covid-19: living systematic review and network meta-analysis. BMJ. $2020 \mathrm{Jul}$ 30;370:m2980.

10. Li YD, Chi WY, Su JH, Ferrall L, Hung CF, Wu TC. Coronavirus vaccine development: from SARS and MERS to COVID-19. J Biomed Sci. 2020 Dec 20;27(1):104.

11. Rhoades RA, Pflanzer RG. Human Physiology, 5th ed. Thomson Learning, 2002.
12. Janeway C. Immunobiology, 5th ed. Garland Publishing, 2001.

13. Vabret N, Britton GJ, Gruber C, Hegde S, Kim J, et al.; Sinai Immunology Review Project. Immunology of COVID-19: Current State of the Science. Immunity. 2020 Jun 16;52(6):910-941.

14. Ledford $\mathrm{H}$. Coronavirus reinfections: three questions scientists are asking. Nature. 2020 Sep;585(7824):168-169.

15. Vergano D. COVID-19 Vaccines Work Way Better Than We Had Ever Expected. Scientists Are Still Figuring Out Why. BuzzFeed News. Retrieved 24 June 2021.

16. Regulatory Decision Summary - PfizerBioNTech COVID19 Vaccine. Health Canada, Government of Canada. 9 December 2020.

17. A Study to Evaluate Efficacy, Safety, and Immunogenicity of mRNA-1273 Vaccine in Adults Aged 18 Years and Older to Prevent COVID-19. Available at: https://clinicaltrials. gov/ct2/show/NCT04470427.

18. Investigating a Vaccine Against COVID-19. Available at: https://clinicaltrials.gov/ct2/ show/NCT04400838.

19. A Study of Ad26.COV2.S for the Prevention of SARS-CoV-2-Mediated COVID-19 in Adult Participants (ENSEMBLE). Available at: https://clinicaltrials.gov/ct2/show/ NCT04505722.

20. Safety of COVID-19 Vaccines. U.S. Centers for Disease Control and Prevention (CDC). 11 February 2020

21. Kowalski PS, Rudra A, Miao L, Anderson DG. Delivering the Messenger: Advances in Technologies for Therapeutic mRNA Delivery. Mol Ther. 2019 Apr 10; 27(4):710-728.

22. Comparing the COVID-19 Vaccines: How Are They Different? Available at: https:// www.yalemedicine.org/news/COVID-19 -vaccine-comparison.

23. Newborns Won't Get COVID Through Infected Mom's Breast Milk: Study. Availale at: https://myhealth.ucsd.edu/ Relatedltems/6,1651376430.

24. Fox A, Marino J, Amanat F, Krammer F, Hahn-Holbrook J, Zolla-Pazner S, Powell RL. Robust and Specific Secretory IgA Against SARS-CoV-2 Detected in Human Milk. iScience. 2020 Nov 20;23(11):101735.
25. Gao X, Wang S, Zeng W, et al. Clinical and immunologic features among COVID-19affected mother-infant pairs: antibodies to SARS-CoV-2 detected in breast milk. New Microbes New Infect. 2020 Sep;37:100752.

26. Kelly JC, Carter EB, Raghuraman N, et al. Anti-severe acute respiratory syndrome coronavirus 2 antibodies induced in breast milk after Pfizer-BioNTech/BNT162b2 vaccination. Am J Obstet Gynecol. 2021 Jul;225(1):101-103.

27. Perl SH, Uzan-Yulzari A, Klainer $H$, Asiskovich L, et al. SARS-CoV-2-Specific Antibodies in Breast Milk After COVID-19 Vaccination of Breastfeeding Women. JAMA. 2021 May 18;325(19):2013-2014.

28. Gray KJ, Bordt EA, Atyeo C, Deriso E, et al. Coronavirus disease 2019 vaccine response in pregnant and lactating women: a cohort study. Am J Obstet Gynecol. 2021 Mar 26:S0002-9378(21)00187-3.

29. COVID mRNA vaccines induce immune response in pregnant, lactating women. Available at https://www.cidrap.umn.edu/ news-perspective/2021/05/covid-mrnavaccines-induce-immune-responsepregnant-lactating-women.

30. After Getting a COVID19 Vaccine, Women Are Selling Their Breast Milk Online. Available at https://www.usnews.com/news/ health-news/articles/2021-04-22/women-areselling-breast-milk-online-after-getting-acovid-vaccine.

31. Götzinger F, Santiago-García B, NogueraJulián A, et al.; ptbnet COVID-19 Study Group. COVID-19 in children and adolescents in Europe: a multinational, multicentre cohort study. Lancet Child Adolesc Health. 2020 Sep;4(9):653-661.

32. Collier AY, McMahan K, Yu J, Tostanoski LH, Aguayo $R$, et al. Immunogenicity of COVID-19 mRNA Vaccines in Pregnant and Lactating Women. JAMA. 2021 Jun 15;325(23):2370-2380.

33. Bertrand K, Honerkamp-Smith G, Chambers C. Maternal and child outcomes reported by breastfeeding women following mRNA COVID-19 vaccination. Available at: https:// www.medrxiv.org/ content/10.1101/2021.04.21.21255841v1. 